\title{
Evaluation of Piezotome-Corticision Assisted Orthodontics in Retracting Upper Canine: A Split Mouth Design (Randomized Clinical Trial)
}

\author{
Dr. Samer Al Refaii \\ Clinical Assistant professor \\ Department of Developmental Sciences \\ Faculty of Dentistry, Beirut Arab University, Lebanon \\ Professor. Nadia EI Harouni \\ Department of Orthodontics \\ Faculty of Dentistry, Beirut Arab University, Lebanon \\ Assistant Prof. Aly Osman \\ Department of Developmental Sciences \\ Faculty of Dentistry, Beirut Arab University, Lebanon
}

\section{Doi:10.19044/esj.2021.v17n34p162}

Submitted: 06 September 2021

Accepted: 17 September 2021

Published: 30 September 2021
Copyright 2021 Author(s)

Under Creative Commons BY-NC-ND 4.0 OPEN ACCESS

Cite As:

Al Refaii S., El Harouni N. \& Osman A. (2021). Evaluation of Piezotome- Corticision Assisted Orthodontics in Retracting Upper Canine: A Split Mouth Design (Randomized Clinical Trial. European Scientific Journal, ESJ, 17(34), 162.

https://doi.org/10.19044/esj.2021.v17n34p162

\section{Abstract}

Objectives: The aim of this randomized clinical trial is to investigate the rate of canine retraction at different time points between piezotomecorticision assisted and conventional orthodontics.

Methods: Fifteen patients were submitted for upper first premolars extraction after leveling and alignment was achieved to facilitate canine retraction. They were randomly divided into test side and control side. On the test side, the canine was retracted using piezotome-corticision assisted orthodontics, while at the control side, the canine was retracted using conventional technique. Both groups were immediately loaded with a horizontal force of $150 \mathrm{~g}$ for canine retraction. The distance between the canine bracket hook and the first molar band hook were recorded using a digital caliper. The measurements were accomplished immediately after piezosurgery at $1,2,4,8$ and 12 week. 
Results: There was a statistically significant difference in the average amount of canine retraction between test and control groups $(\mathrm{P}<0.05)$. The canine on the test side was closed 6 weeks earlier than the control side.

Conclusion: Piezocision is minimal invasive which accelerate the rate of canine of retraction approximately 1.5 times faster than that of conventional method.

Keywords: Piezotome, Corticision Assisted Orthodontics, Acceleration

\section{Introduction}

Prolonged treatment time is considered a drawback since it is usually associated with poor patient compliance, caries, periodontal disease and also root resorption may occur. Thus, reducing orthodontic treatment time is one of the primary goals for orthodontists as it leads to increased patient satisfaction and acceptance. The patients have specific demands and usually want to achieve their treatment goals as soon as possible to reduce the negative effects of orthodontic appliances in their social and professional lives. Since orthodontic tooth movement (OTM) is caused by a gradual remodeling (apposition and resorption cycle) of supporting alveolar bone, the factors affecting this cycle could control the rate of tooth movement (Norevall et al 1995).

Therefore, the acceleration of the orthodontic tooth has become one of the primary concerns among orthodontists worldwide. Several methods have been proposed to shorten the treatment time, such as the use of low friction self-ligating brackets (SLBs), pharmacological approaches, physical stimuli, and surgical methods (Nimeri et al 2013).

The surgical interventions have been found to be the most effective in enhancing the tooth movement and the most widely used, with predictable outcomes (Alfawal et al 2016).

These surgical interventions include conventional corticotomy, interseptal alveolar surgery, accelerated osteogenic orthodontics, dentoalveolar distraction, and periodontal distraction (Patterson et al 2016).

Although the conventional corticotomy procedures have been proven to be effective in reducing the orthodontic treatment time, they have adverse sequelae, such as interdental bone loss, loss of the attached gingiva, periodontal defects, and hematomas in the neck and face. These documented complications are due to the invasiveness of the traditional procedures with the need for elevating full thickness periosteal flaps (AlGhamdi 2010). Consequently, several researchers have tended to investigate less invasive surgical acceleration modalities, such as laser-assisted flapless corticotomy, piezocision and micro osteoperforations (Alfawal et al 2016). 
Piezocision has recently evolved as a novel approach of manipulating the cortical bone with minimal damage, less discomfort and greater patient acceptance (Charavet 2016). Dibart et al. (2009) used a piezoelectric knife to achieve flapless alveolar decortication, subsequently inducing regional acceleration phenomenon (RAP) with the possibility of hard or soft tissue grafting, using selective tunneling procedures.

The acceptance of traditional corticotomy-assisted orthodontics among patients was generally low, mainly because of the invasive procedures and postoperative discomfort and complications (Cassetta 2012). Flapless piezocision-assisted corticotomy has been found to have various advantages over the traditional methods of corticotomy and is considered a promising minimally invasive tooth acceleration technique (Alfawal 2016).

Therefore, we promoted this study to evaluate the time required to achieve a canine retraction between piezotome-corticision assisted and conventional orthodontics.

\section{Materials and Methods}

Study design and setting: This study was carried out as an experimental, randomized controlled clinical trial (split mouth design). The patients were selected conveniently from the outpatient clinic at Beirut Arab University treated at the speciality clinics.

The estimated sample size was calculated according to http://epitools.ausvet.com.au, by taking the means and the variance of a similar study conducted by Naoum et al (2014) where the mean for the test side was 0.739 and the mean for the control side was 0.201 and the variance was 0.133 , the confidence level was set to $95 \%$ and the power was set on $80 \%$. The calculated sample size was 14 patients. Therefore, 15 patients (30 operating sides) who required for first premolar extraction were recruited from the outpatient clinics Department of Orthodontics, Faculty of Dentistry. inclusion criteria included patients of age ranged from 18-30 years having Class II division 1, in which extraction of upper first premolars were required. Also patients with good oral and gingival health and with full set of teeth in the maxillary arch till the second molar.

The Ethical Committee (Institutional Review Board, IRB number: 2015H-0027-D-P-97 gave the ethical clearance before the study began, the selected patients were informed about the nature of the study, and informed consent was obtained. 


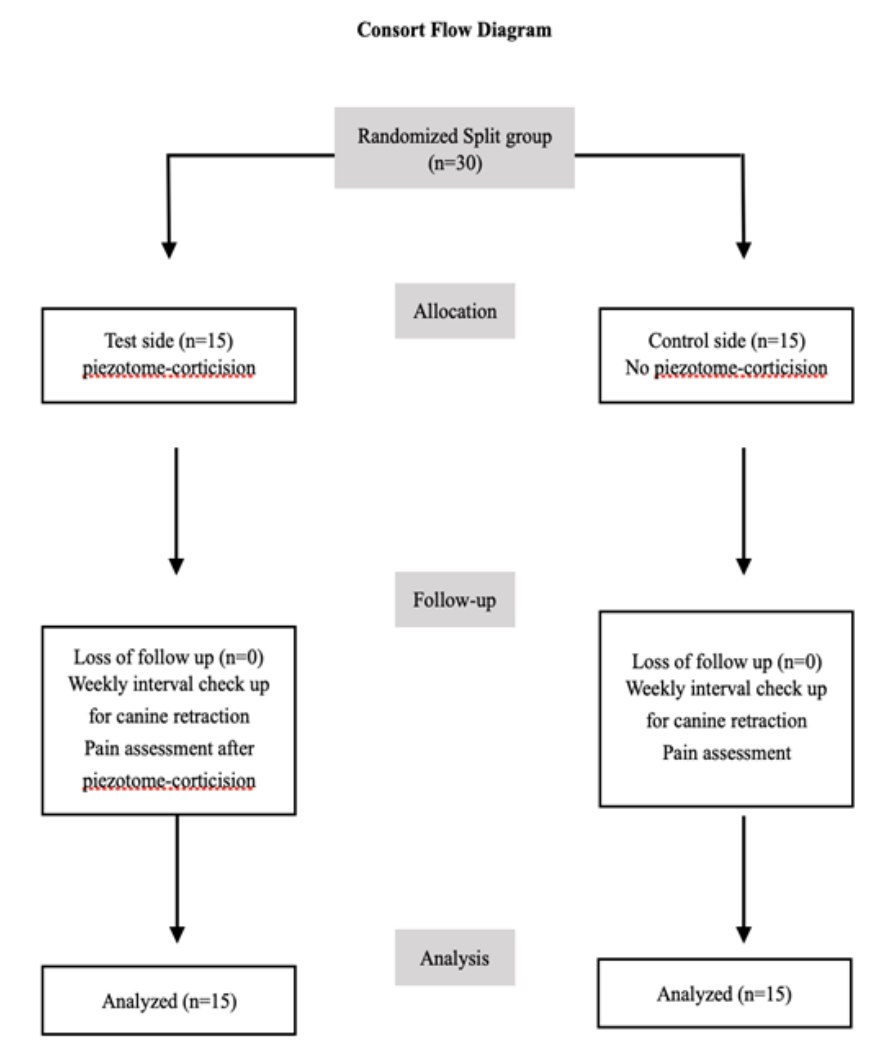

Fig.1. Consort Flow

\section{Method}

\section{Diagnostic phase}

The diagnostic records were collected from each patient including study casts, photographs, lateral cephalometric and panoramic radiographs. The transpalatal arches were cemented to avoid the mesial movement of the first molar. After finishing the leveling and aligning of the upper arch and reaching stiff arch wire (0.16x0.22 stainless steel), the second premolars and the first molars and second molars were lighted and then the 2 upper first premolars were extracted by an oral surgeon.

The study was a split mouth design, each patient had a test side (T) and the control side (C). The randomization was performed of the selected side by tossing a coin, the face was the test side $(\mathrm{T})$; canine was retracted using piezotome-corticision assisted orthodontics and the back was the control side (C); canine was retracted using conventional orthodontics. 


\section{Surgical phase}

The patients were asked to rinse with mouth wash before the surgical intervention. Local anesthetic was injected using Lidocaine hydrochloride $2 \%$. The local anesthesia was administrated through infiltration beside the upper canine. The depth of gingival tissue was checked by bone sounding using a Williams periodontal probe. Panoramic X-rays were employed to assess the long axis of the teeth and root proximity prior to the procedure.

The study sides underwent the piezotome-corticision procedure after one month of extraction of upper first premolar. This procedure was performed by the technique explained by Kesser et al (2011). 15C Bard-Parker blade was used to make incisions through the gingiva, $4 \mathrm{~mm}$ above the interdental papilla to save the coronal attached gingiva. The incisions were $4 \mathrm{~mm}$ in length mesial and distal to the upper canine. After the incisions were made, the gingiva was slightly elevated laterally by the aid of periosteal elevator to visualize the bone.

A piezosurgery insert (OT7) which is an ultrasonic microsaw, was used to create the cortical alveolar incisions to a depth of $1 \mathrm{~mm}$ within the cortical bone. The vertical level of the measurement was established to be $4 \mathrm{~mm}$ apical to the crest of the alveolar bone (fig.2).

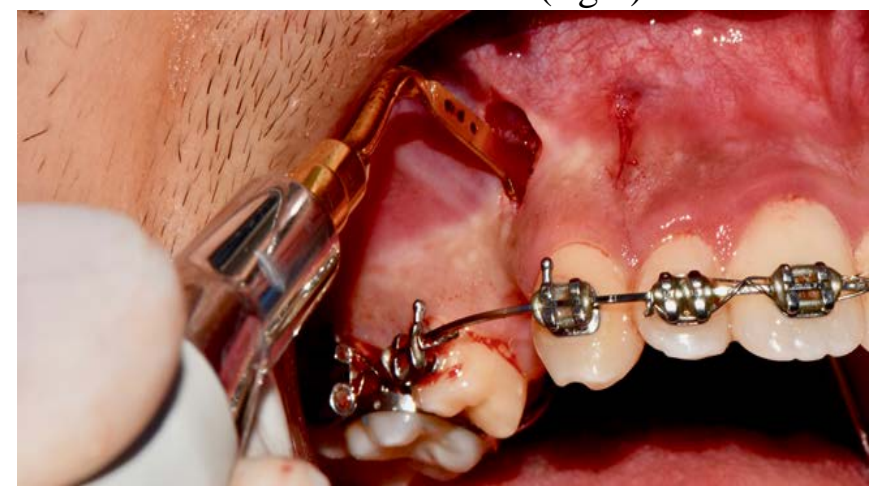

Fig.2. Cortical incision using piezosurgery inserts OT7

\section{Force Application}

Immediately after the piezosurgery, the canines of the experimental and control sides were moved distally along the orthodontic wire with a continuous force of $150 \mathrm{~g}$ using nickel titanium closed coil springs on $0.016 \times$ 0.022-in stainless steel arch wires (Kazuo et al 2004). One end of the spring was fixed to the hook of the canine bracket with a ligature wire, and the other side was fixed to the hook of the band of the upper first molar (fig.3). 


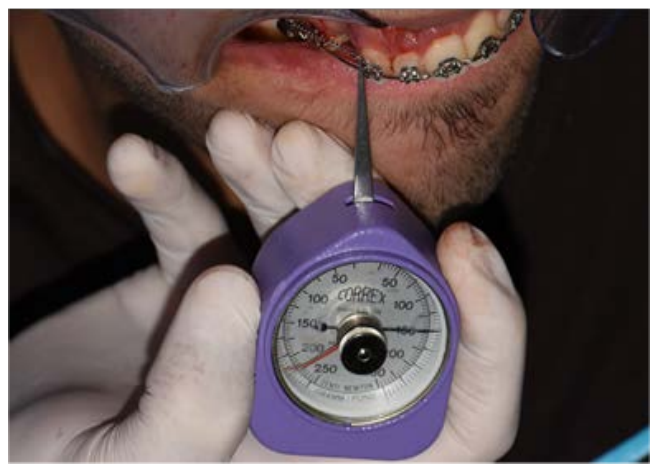

Fig.3. Measuring the force magnitude for canine retraction using force gauge

\section{Clinical Evaluation}

The distance between the canine bracket hook and the first molar hook was recorded using a digital caliper (Fig.4) The measurements were accomplished immediately after piezosurgery and at 1, 2, 4, 8, and 12 weeks after piezosurgery till complete retraction of canine. These measurements were considered an indicator of canine retraction speed (Naoum et al 2014).

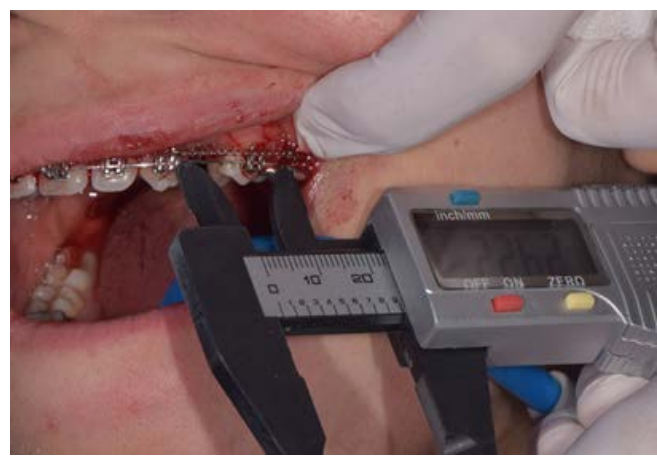

Fig.4. Closed NiTi coil spring for retraction of canine

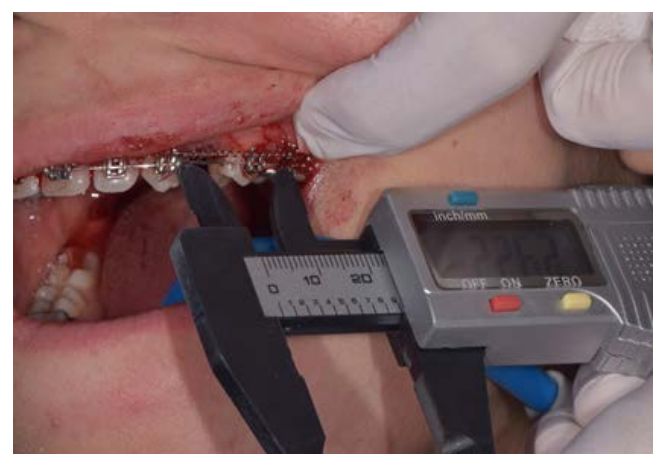

Fig.5. Measuring the distance of the canine retraction

\section{Statistical analysis}

The mean and standard deviation of the various variables were calculated. To calculate the change from one time point to the next, the values were subtracted. Normality was checked using Kolmogorov Smirnov test. Differences were compared between groups using paired t test and Wilcoxon signed ranks test as indicated. $\mathrm{P}<0.05$ was considered to be statistically significant. Statistical analysis was conducted using SPSS version 22.0.

\section{Results}

This study was conducted as a randomized controlled clinical trial: split mouth design. Fifteen patients with a mean age of 24 years, who required 
bilateral upper first premolar extraction from the in-patient clinic of the Orthodontic Department, Faculty of Dentistry, Beirut Arab University.

Table 1 . shows the descriptive statistics of the average amount of canine movement on the experimental side, compared to that on the control side over different time periods. The table shows a statistically significant difference in the average amount of canine movement between both groups throughout the study with increased tooth movement on the test side compared to the control.

The greatest average distance moved by canine at the experimental side had been recorded at week $1(0.86 \pm 0.09 \mathrm{~mm})$. (fig.6)

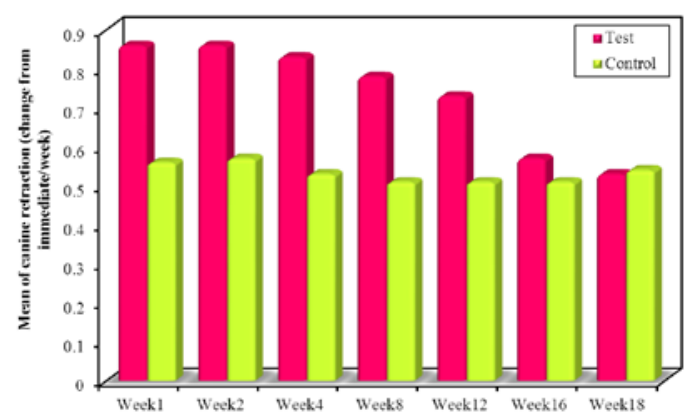

Fig. 6. Comparison between the studied groups according to Canine retraction

Table 2. shows that the mean weekly retraction in the test side was greater than in the control side between T0-T1, T1-T2, T2-T4, T4-T8, and T8-T12 with mean difference $=0.3,0.28,0.31,0.25$, and 0.13 . The difference between the two groups are statistically significant throughout the experiment. The tooth velocity was found approximately 1.5 times faster on the experimental side (Fig.7).

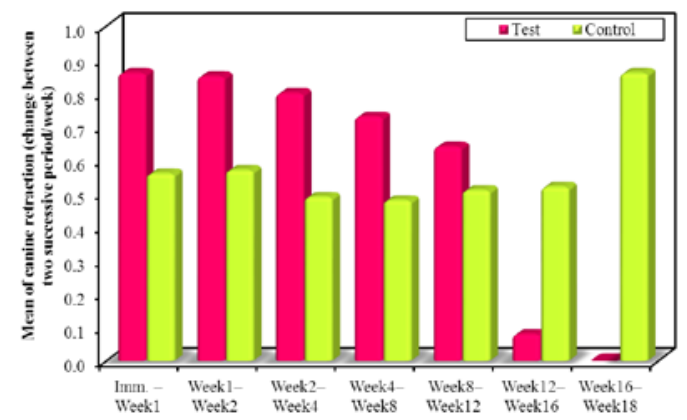

Fig.7. Comparison between the studied groups according to Canine retraction

\section{Discussion}

The treatment time is an important issue for orthodontic patients. In recent decades, major efforts have been made to shorten the length of treatment to minimize the complications and to increase satisfaction of the 
patient. Multiple approaches have been demonstrated to enhance the rate of orthodontic tooth movement.

The combination of orthodontics and corticotomies, showed a positive power in terms of reducing overall orthodontic treatment times (Wilcko et al 2009). However, these techniques have not been widely embraced by the dental community since they require extensive full thickness flap elevation and in cases of osteotomy, an invasive procedure associated with postoperative discomfort and a high risk of complications such as possible damage to teeth and bone, possible marginal osteonecrosis risk and impair bone regeneration leading to a low acceptance by the patient (Kim et al 2009).

Piezoelectric incisions were now suggested because of their safety and effectiveness in different types of surgeries (Vercellotti 2000). Similar clinical outcomes were demonstrated between piezocision and classical decortication but piezocision has the added advantages of being quick, minimally invasive, and less traumatic to the patient.

The aim of this study was to evaluate time required to achieve a canine retraction between piezotome-corticision assisted and conventional orthodontics.

The rate of canine retraction in the current study were significantly higher in the experimental side than in the control side. This acceleration might be clarified by the RAP phenonomena and due to the reduction of alveolar bone resistance to tooth movement (Wilko et al 2001). As well as due to the stimulation of the expression of the inflammatory markers and the increase the levels of cytokines that lead to promote the activity of osteoclasts which in turn motivate bone remodeling and acceleration of tooth movement (Baloul et al 2011).

The maximum rate of canine retraction on the test side $(0.86$ $\mathrm{mm} /$ week) was observed in the first and the second weeks then it began to decrease gradually there after and showed a sharp drop after the 12th week. Wilcko et al (2009) stated that this phenomena continues for 2 to 4 months. This difference between the present findings and those of Wilcko et al could be attributed to the more aggressive nature of their technique compared to that of the current minimal invasive technique. They demonstrated that when the bone injury is severe, this is directly correlated to the intensity of bone healing and the rapidity of tooth movement. The increased rate of canine retraction on the test side compared to the control group was evident in the first 12 weeks as afterwards most of the canines at the test side were completely retracted, so the movement dropped to zero.

The overall rate of canine retraction on the test side was significantly higher by1.5 times than that on the control side which agrees with the findings of Aksakalli et al (16). 
Also these findings agree with Abbas et al (17), who revealed that piezocision was able to accelerate the rate of canine retraction by 1.5-2 times during the first 3 months of tooth movement. The current study was relatively close to those done by alikhani et al (2013), who clinically tested the effect of microosteoperforations on the velocity of canine retraction and stated that the rate of the tooth movement on the test side was greater by 2.3 fold than that on the control side.

In agreement with our study, Omar et al (2018) compared flapless piezocision-assisted corticotomy in the extraction-based orthodontic decrowding of lower anterior teeth with the conventional treatment. The study concluded that flapless piezocision technique was very effective in accelerating orthodontic tooth movement.

Moreover, Ghaith et al (2019) conducted a randomized controlled clinical trial was to assess the effectiveness of the flapless piezocision procedure in accelerating the retraction of upper incisors. He concluded that piezocision procedure an effective in accelerating the retraction of 4 upper incisors, reducing the retraction time, preserving anchorage and enhancing root torque control during retraction.

\section{Conclusions}

- The piezocision is an innovative, minimal invasive, flapless procedure and less traumatic for the patient. This method has been proven to facilitate the acceleration of tooth movement.

- The piezotome-corticision technique accelerated the rate of canine retraction approximately 1.5 times faster on the test side.

\section{References:}

1. Aksakalli S, Calik B ,Kara B, Ezigganli S.Accelerated tooth movement with piezocision and its periodontal-transversal effects in patients with class II malocclusion. Angle Orthod.2016;86:59-65.

2. Alfawal AM, et al. Effectiveness of minimally invasive surgical procedures in the acceleration of tooth movement: a systematic review and meta-analysis. Prog Orthod. 2016;17:33.

3. AlGhamdi AST. Corticotomy facilitated orthodontics: Review of a technique. Saudi Dent J. 2010;22(1):1-5.

4. Baloul, S.S., et al., Mechanism of action and morphologic changes in the alveolar bone in response to selective alveolar decorticationfacilitated tooth movement. Am J Orthod Dentofacial Orthop 2011;139(4): S83-101.

5. Cassetta M, et al. The impact of osteotomy technique for corticotomyassisted orthodontic treatment (CAOT) on oral health-related quality of life. Eur Rev Med Pharmacol Sci. 2012;16:1735-40. 
6. Charavet C, et al. Localized piezoelectric alveolar decortication for orthodontic treatment in adults: a randomized controlled trial. J Dent Res. 2016;95:1003-9.

7. Dibart S, Sebaoun JD, Surmenian J. Piezocision: A Minimally Invasive, Periodontally Accelerated Orthodontic Tooth Movement Procedure. Compend Contin Educ Dent. 2009 Jul-Aug;30(6):342-4, 346, 348-50.

8. Ghaith M.F. Al-Imam, Mowaffak A. Ajaj, Mohammad Y. Hajeer, Yaser Al-Mdalal, Eyad Almashaal. (2019) Evaluation of the effectiveness of piezocision-assisted flapless corticotomy in the retraction of four upper incisors: A randomized controlled clinical trial. Dent Med Probl.;56(4):385-394

9. Kesser EI, Dibart S. Piezocision-Assisted Invisalign Treatment. Compend Contin Educ Dent. 2011 Mar; 32(2): 46-8, 50-1.

10. Naoum, Hajeer, and Al-Jundi. Acceleration of Tooth Movement by Alveolar Corticotomy. J Oral Maxillofac Surg 72:1880-1889, 2014.

11. Nimeri G, Kau CH, Abou-Kheir NS, Corona R. Acceleration of tooth movement during orthodontic treatment $-\mathrm{a}$ frontier in orthodon- tics. Prog Orthod. 2013;14:42.

12. Norevall L.I, Forsgren S, Matsson L. Expression of neuropeptides (CGRP, substance P) during and after orthodontic tooth movement in the rat. Eur J Orthod, 1995. 17(4): 311-25.

13. Omar Gibreal1, Mohammad Y. Hajeer2 and Bassel Brad3. (2018) Efficacy of piezocision-based flapless corticotomy in the orthodontic correction of severely crowded lower anterior teeth: a randomized controlled trial. European Journal of Orthodontics, 2018, 1-8 doi:10.1093/ejo/cjy042

14. Patterson BM, Dalci O, Darendeliler MA, Papadopoulou AK. Corticotomies and orthodontic tooth movement: A systematic review. J Oral Maxillofac Surg. 2016;74(3):453-473.

15. Vercellotti T. Piezoelectric surgery in implantology: a case report-a new piezoelectric ridge expansion technique. Int $\mathrm{J}$ Periodontics Restorative Dent. 2000;20(4):358-365.

16. Wilcko T, Wilcko M. Accelerated Osteogenic Orthodontics Technique: A 1-Stage Surgically Facilitated Rapid Orthodontic Technique With Alveolar Augmentation. J Oral Maxillofac Surg 67:2149-2159, 2009.

17. Wilcko W.M, et al. Rapid orthodontics with alveolar reshaping: two case reports of decrowding. Int J Periodontics Restorative Dent, 2001. 21(1): 9-19. 